\title{
A Service Management Architecture for NEMO in IPv4 and IPv6 Networks ${ }^{\star}$
}

\author{
Jin Ho Kim, Choong Seon Hong ${ }^{\star \star}$, Dae Sun Kim \\ Department of Computer Engineering, Kyung Hee University, \\ Seocheon, Giheung, Yongin, Gyeonggi, 449-701, Korea \\ jhkim@networking.khu.ac.kr, cshong@khu.ac.kr, dskim@networking.khu.ac.kr
}

\begin{abstract}
The current design of Mobile IPv4 and Mobile IPv6 protocols doesn't provide the compatibility between networks of different versions. A Network Mobility(NEMO) protocol also provides the limited mobility in IPv6 networks. However, we can easily expect that the current IPv4 network will coexist with IPv6 network in the near future internet environment. So, in this paper, we propose the architecture of mobility between networks of different versions which is supported the NEMO basic protocol. This new mechanism can manage the mobility through a Tunnel Agent(TA). And the TA maintains a NEMO tunnel when a Mobile Router(MR) moves from IPv6 to IPv4 network.
\end{abstract}

\section{Introduction}

With the rapid development of the radio communication technology, users tend to exchange all kinds of data through wireless internet. It increases the desire that users want to access the internet from anywhere, at anytime and the connection is expected to be seamless. As the solutions for these requirements, Internet Engineering Task Force Working Group(IETF WG) made an effort to standardize the method to support mobility of terminals. In particular, the specification of the Mobile IPv6[1] has been standardized as RFC 3775. Recently, the support for mobility of network such as buses, airplanes, cars and trains as well as that of terminal unit is discussed actively. Discussion about supporting network mobility method is progressing vigorously in IETF NEMO WG[2]. First, NEMO WG established the extended Network Mobility(NEMO) basic protocol[3] from the Mobile IPv6 in RFC 3963. It can offer the seamless connectivity of internet for terminals in mobile network. Furthermore, they have plans to standardize the extended NEMO such as route optimization and multihoming.

The most distinguished feature of network mobility is that the mobile router, not each node, is wholly responsible for maintaining the mobility. In other words, whenever the point of internet connection is changed, only one signal of the MR can replace several signals processed by each terminal. So, the signaling overhead of network is decreasing. Also, usual IPv6 appliances which don't support IP mobility can still use the internet even if the internet connection point is changed. But, if the introduction of IPv6 can't provide interoperability with

\footnotetext{
* This work was supported by MIC and ITRC Project.

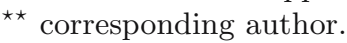


existing IPv4 environment, it isn't useful. Therefore, conversion technologies between IPv4 and IPv6 are essential. IETF Network Generation Transition Working Group(NGTrans WG) had already established transition technologies of dual stack, tunneling, translator for fixed hosts as its standard. But, mobility support isn't considered in these mechanisms.

If session connectivity is supported, mobile nodes and MNNs(Mobile Network Nodes)[4] can move from IPv6 to IPv4 network. But, the session connectivity for supporting mobility in the protocols such as Mobile IPv6 and NEMO isn't guaranteed because it isn't compatible with the networks of different versions. Therefore, in this paper, we propose the architecture that guarantees to retain session connectivity of the NEMO tunnel by the Tunnel Agent(TA) in case where mobile network moves to the IPv4 network.

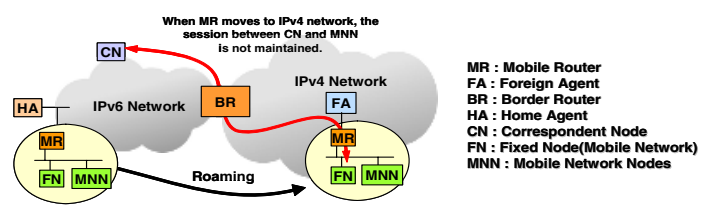

Fig. 1. Roaming scenario of Mobile Network

This paper is organized as follows. Section 2 introduces some related works such as dual stack, Doors mechanisms and IPv4 Care-of Address(CoA) Registration scheme. In section 3, we describe the operation of the TA and the MR. Then we show the result of performance evaluations in section 4. Finally, we conclude in section 5 .

\section{Related Works}

The simplest mechanism for transition between Mobile IPv4[5] and Mobile IPv6 is a dual stack structure[6]. But, a designer must implement the dual stack of the Mobile Node(MN) supporting each version of two mobility protocols. When the terminal moves to other links, it needs to send signal to the Home Agent(HA)[1][3][5] of IPv4 and IPv6 networks to keep connectivity. For example, when terminals move to the network of another version, they have the burden which must process all Registration Request[5] and Registration Reply[5] messages in Mobile IPv4, Binding Update(BU)[1] and Binding Acknowledgement(BA)[1] messages in Mobile IPv6.

Doors mechanism[7] suggests using a 'Doors Router' between IPv4 and IPv6 networks. In this mechanism, when an MR moves to a Private $\mathrm{IPV}_{\mathrm{v}}$ network as well as a Public IPv4 network, sessions of each network are maintained. But, when the MR roams to the IPv4 network, MNNs carry tunneled packet of IPv4IPv6-IPv6 headers to an HA. Then tunnel overhead increases additionally.

IPv4 CoA Registration mechanism[8] is introduced to reduce tunnel overhead compared with tunnel in the Doors mechanism. When the MR moves to IPv4 network, it adds an option including the IPv4 CoA in the BU message. And the 
$\mathrm{MR}$ registers the $\mathrm{IP}_{\mathrm{v}} 4 \mathrm{CoA}$ of the $\mathrm{MR}$ to the $\mathrm{HA}$ which carries packets with a reduced IP header, IPv4-IPv6, to the MNNs. So, the tunnel overhead decreases.

\section{Proposed Architecture}

This paper proposes an architecture for supporting mobility through a TA when an MR including MNNs move to an IPv4 network. That is, as the TA relays tunnel end point of IPv6 and IPv4 networks, a NEMO tunnel is kept to work. Basically, the TA and the MR are dual stack(IPv4/IPv6) structure, and the MR must include the minimal Mobile IPv4 protocol function which can process an Agent Advertisement[5] message to compose an IPv4 CoA in the IPv4 network environment. In other words, the signaling overhead of the proposed architecture decreases as compared to the other proposed scheme using the dual stack which has all functions of Mobile IPv4 and Mobile IPv6 protocols.

Obtaining the Tunnel End Point Address. We assume that an HA already knows TA's IPv4/IPv6 addresses. For instance, when the HA gets tunnel end point address automatically, it gets IPv4/IPv6 addresses of the TA in current network using DSTM[9]. But, if the HA cannot use the DSTM, it can use configured address. Figure 2 is a proposed message format of Home Agent Address Discovery Request/Reply[3] messages which has a "V" flag and a Tunnel Agent Information(TAI) option additionally. If the MR moves to the IPv4 network, and wants to maintain its session from the TA, it sets the "V" flag and sends the Home Agent Address Discovery Request message by an Anycast address. Then, the HA knows the tunnel end point address and responds the Home Agent Address Discovery Reply message which includes the TAI option with the "V" flag to the MR. At this time, the TAI option must include the TA's IPv4/IPv6 addresses necessarily, and the MR must store this information.

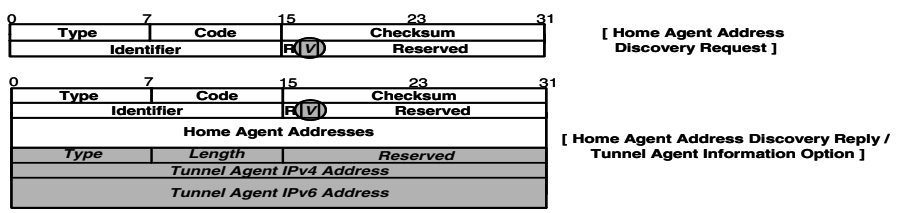

Fig. 2. Home Agent Address Discovery Request/Reply and Tunnel Agent Information(TAI) option message format

Movement Detection of IPv4 Networks and Home Registration. If we use the mechanism defined in the NEMO basic protocol standard, mobility of the MNNs in the MR which roams only in the IPv6 network can be supported without any problem. If the MR already knows the TA's address through Home Agent Address Discovery mechanism[3], mobility can be supported in the IPv4 network. This paper assumes that the MR has dual stack and has the minimum functionality to compose the IPv4 CoA for detecting movement in the IPv4 


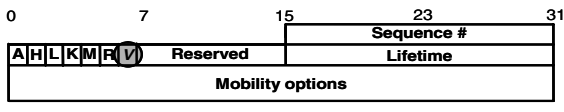

[ Binding Update Message with "V" Flag ]

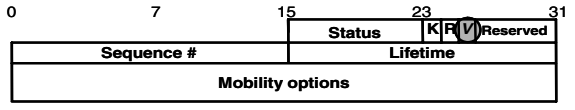

[ Binding Acknowledgement Message with "V" Flag ]

Fig. 3. Binding Update/Acknowledgement messages format

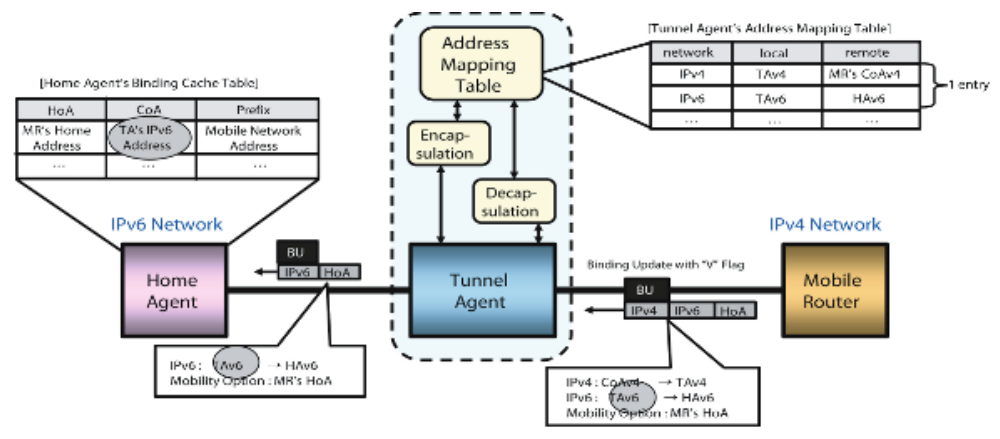

Fig. 4. Home Registration process and Tunnel Agent architecture

network. That is, the MR can detect movement in the IPv4 network by receiving the Agent Advertisement message and compose the IPv4 CoA.

If the MR detects its movement in the IPv4 network, then the MR sends the BU message with the "V" flag to the HA in order to register the information. Figure 3 shows BU and BA messages format having the "V" flag.

Figure 4 shows the address mapping table of the TA, the HA's binding cache table architecture and the BU message format which the MR sends to the HA. The MR composes the source address of the BU message as own CoA in the NEMO basic protocol. But, in this paper, when the MR moves to the IPv4 network, it sends the BU message to the HA, its source address is decided by the tunnel end point's IPv6 address which is known by the MR already. And the MR sends the encapsulated the IPv4 header to the TA. In other words, an IPv4 protocol number in the BU header field must be 41 to inform that it is the IPv6 packet, source address is the MR's IPv4 CoA, and destination address is the TA's $\mathrm{IPv} 4$ address. After the TA receives the BU message, it decapsulates the IPv4 header and forward it to the HA. The HA receives the BU from the TA registers the MR's CoA moving to the IPv4. Therefore, as the source address of the BU message is the TA's IPv6 address, the HA makes the MR's IPv6 CoA to the TA's IPv6 address and updates binding cache[3]. The HA sends all packets to the TA while the MR moves to the IPv4 network. The reason is that the CoA which is mapped to an MR's home address[3] in binding cache had the TA's IPv6 address.

NEMO Tunnel Setup. If BU and BA messages are exchanged successfully between the HA and the TA, the NEMO tunnel is established. In the NEMO basic protocol, when the MNNs communicate with a Correspondent $\operatorname{Node}(\mathrm{CN})$, packets are always transmitted through an HA-MR tunnel[3]. Therefore, it is the most important that the NEMO tunnel is maintained. In case the MR moves 
to the IPv4 network, because an HA-MR tunnel cannot be kept continuously, the TA must manage tunnel cache so that NEMO tunnel between the HA and the MR can be kept. In dual stack structure and Doors mechanism, three IP headers, IPv4-IPv6-IPv6 are created. But, in this paper, the MR establishes an efficient a TA-MR(IPv6 in IPv4) tunnel considering handoff to other links in the IPv4 network. The HA makes a IPv6-IPv6 tunnel between the HA and the TA because the MR's CoA is established to the TA's address. And the TA finishes the home registration processes of the MR after creating a IPv4-IPv6 tunnel with the MR. If the MR moves to another link within the $\mathrm{IPv} 4$ network, the $\mathrm{MR}$ sends the BU message and the TA receives this message. Then we need to only set the TA-MR tunnel. That is, only the TA's tunnel end point is changed and the HA-TA tunnel(IPv6-IPv6) isn't changed. Therefore, additional signaling between the TA and the HA can be reduced.

CN Communicates with MNN. When the CN communicates with the MR's MNNs which move to the IPv4 network, packets are transmitted like figure 5 . First, if the CN sends a packet to the MNNs, the HA intercepts the packet because the destination address is an MR's mobile network prefix[4]. After searching the binding cache entry, the HA forwards the packet to the TA using the HA-TA tunnel because registration of the MR's IPv6 CoA to the TA's IPv6 address. If the TA receives the packet from the HA, it transmits the packet to the MR's IPv4 CoA which is the tunnel end point of the TA-MR tunnel. Simultaneity, the TA searches mapping table and translates IPv6 and IPv4 headers. This process uses methods such as header translation mechanism of NAT-PT[11] and SIIT[12]. If the MR receives all packets through the TA-MR tunnel it decapsulates a packet of them for removing its $\mathrm{IPv} 4$ header. Finally, the MR forwards the packet to the MNNs. On the contrary, the packet transmitted from the MNNs to the $\mathrm{CN}$ is the format that the source address is changed to the destination address as figure 5 shows.

IPv4 CoA De-registration. If the MR returns from the $\mathrm{IPV}_{\mathrm{v}} 4$ network to the IPv6 network, it sends the BU message of which the "V" flag isn't sets to the TA. After that, the TA must release the TA-MR tunnel and remove the tunnel cache. And the TA forwards the BU message to the HA. If the HA receives the $\mathrm{BU}$ for de-registration, it updates the CoA with actual the MR's CoA instead

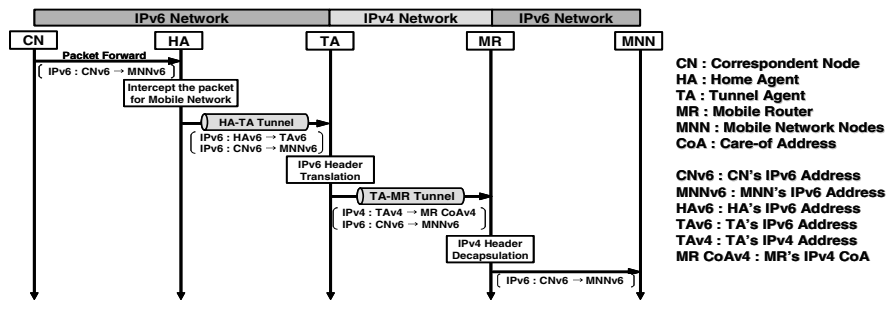

Fig. 5. Communication process flowchart between $\mathrm{CN}$ and $\mathrm{MNN}$ 
of the TA's address in the binding cache. The handoff in IPv6 network is similar to the operation defined in the NEMO basic protocol standard. In other words, communication between the MNNs and the CN uses the HA-MR tunnel.

\section{Performance Measures and Analysis}

We can show that the proposed architecture is efficient by measuring the handoff signaling and the packet delay. Figure 6 is the network topology for measurement of the delay. Figure 6(a) shows one scenario of the MR's handoff in an $\mathrm{IPv}_{4}$ network after the node roams from the IPv6 to the IPv4 network. And figure 6(b) shows another scenario that MNNs which move to the IPv4 network communicates with the network which includes an HA and a CN in other IPv6 networks. The following figure 6(c) and equation is referred from [12]. Figure 6(c) shows parameters that indicate meaning and value of each symbol for measuring the performance.

Handoff Signaling Delay. As the MR goes away from the HA in the IPv4 network, an HD measures the value of handoff delay. In other words, we calculate the handoff delay as the hop count is increasing in the IPv4 network(The hop count of the IPv6 network isn't changed). The delay of handoff signaling in the two mechanisms is shown in figure 6(a):

- In Doors mechanism, the MR should communicate BU/BA messages with the own the HA directly when it handoffs in the IPv4 network.

$$
\begin{aligned}
H D= & 2\left(\frac{L_{F A-M R} \cdot H_{4-M}}{B W_{\text {wired }}}\right)+\frac{\left(L_{T A-H A}+L_{H A-T A}\right) \cdot H_{6-H}}{B W_{\text {wired }}}+2\left(\frac{L_{F A-M R}}{B W_{\text {wireless }}}\right) \\
& +4 L_{\text {wired }}+2 L_{\text {wireless }}
\end{aligned}
$$

- Because a TA handles BU/BA messages in the handoff of the proposed architecture, it is more efficient than the Doors mechanism.

$$
H D=2\left(\frac{L_{F A-M R} \cdot H_{4-M}}{B W_{\text {wired }}}\right)+2\left(\frac{L_{F A-M R}}{B W_{\text {wireless }}}\right)+2 L_{\text {wired }}+2 L_{\text {wireless }}
$$

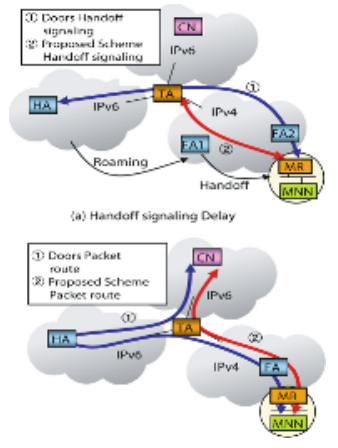

(b) The End-to-End Packet Delay

\begin{tabular}{|l|l|c|}
\hline Symbol & \multicolumn{1}{|c|}{ Meaning } & Value \\
\hline$L_{C N-T A}$ & IP header + payload length of the packet from CN to TA & \\
\hline$L_{T A-H A}$ & IP header + payload length of the packet from TA to HA & \\
\hline$L_{H A-T A}$ & IP header + payload length of the packet from HA to TA & \\
\hline$L_{T A-F A}$ & IP header + payload length of the packet from TA to FA & \\
\hline$L_{F A-M R}$ & IP header + payload length of the packet from FA to MR & \\
\hline$L_{M R-M N N}$ & IP header + payload length of the packet from MR to MNN & \\
\hline$B W_{\text {wired }}$ & Bandwidth of wired links & $100 \mathrm{Mb} / \mathbf{s}$ \\
\hline$B W_{\text {wirless }}$ & Bandwidth of wireless links & $11 \mathrm{Mb} / \mathrm{s}$ \\
\hline$L_{\text {wired }}$ & Propagation delay + link layer delay of wired links & $\mathbf{0 . 5} \mathbf{~ m s}$ \\
\hline$L_{\text {wireless }}$ & Propagation delay + link layer delay of wireless links & $2 \mathrm{~ms}$ \\
\hline$H_{6-H}$ & Number of hops belong to HA's IPv6 Networks & \\
\hline$H_{6-C}$ & Number of hops belong to CN's IPv6 Networks & \\
\hline$H_{4-M}$ & Number of hops belong to MR's IPv4 Networks & \\
\hline
\end{tabular}

(c) Parameters for measuring performance

Fig. 6. Network topology, scenario and parameters for delay evaluation 


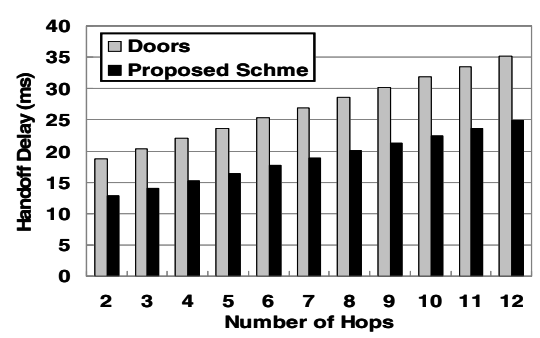

(a) Handoff Signaling Delay

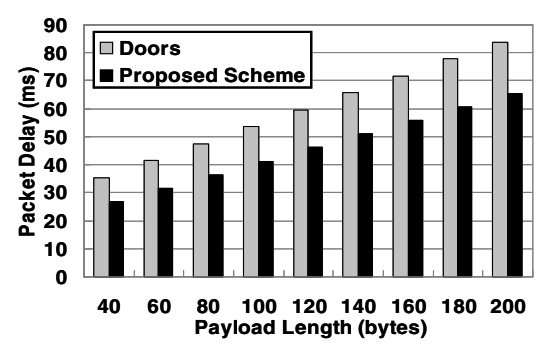

(b) The End-to-End Packet Delay

Fig.7. Performance measures and analysis

Figure 7(a) shows the result of handoff signaling delay. It means that the proposed architecture provides fast handoff when the MR moves within the IPv4 network.

End-to-End Packet Delay. A PD measures the values of the packet delay when the CN communicates with the MNNs in the MR which roam from the IPv6 to the $\mathrm{IPv}_{\mathrm{v}}$ network. We calculated the values of the packet delay by increasing the payload length. The delay of end-to-end packet in the two mechanisms is the shown in figure $6(\mathrm{~b})$ :

- In Doors mechanism, the packet delay of the IPv6 network which includes the HA is occurred because the MNNs communicates with the CN through the HA.

$$
\begin{aligned}
P D= & \frac{L_{C N-T A} \cdot H_{6-C}}{B W_{\text {wired }}}+\frac{\left(L_{T A-H A}+L_{H A-T A}\right) \cdot H_{6-H}}{B W_{\text {wired }}}+\frac{L_{T A-F A} \cdot H_{4-M}}{B W_{\text {wired }}} \\
& +\frac{L_{F A-M R}+L_{M R-M N N}}{B W_{\text {wireless }}}+4 L_{\text {wired }}+2 L_{\text {wireless }}
\end{aligned}
$$

- The proposed architecture forwards packets to the MR directly through a TA-MR tunnel without relaying. So we can reduce the packet delay in the IPv6 network which includes the HA because the packet cannot be carried to the HA.

$$
\begin{aligned}
P D= & \frac{L_{C N-T A} \cdot H_{6-C}}{B W_{\text {wired }}}+\frac{L_{T A-F A} \cdot H_{4-M}}{B W_{\text {wired }}}+\frac{L_{F A-M R}+L_{M R-M N N}}{B W_{\text {wireless }}} \\
& +2 L_{\text {wired }}+2 L_{\text {wireless }}
\end{aligned}
$$

Figure 7(b) shows the result of the end-to-end packet delay. We can see that packet delay decreases as the packet length increases in the proposed architecture compared with Doors mechanism.

\section{Conclusion and Future Work}

In this paper, we proposed an efficient method to support the seamless mobility to IPv4 network through a TA in NEMO protocol supporting the mobility only in IPv6 network. We reduced the signaling overhead by minimizing the Mobile IPv4 protocol stack which compared with dual stack mechanism. When a CN carries 
packets to the IPv4 network, the tunnel overhead is decreased by using IPv4-IPv6 headers through a TA-MR tunnel. Also, we decreased additional binding message between HAs by registering to the TA. Results of Performance measurement show that the proposed mechanism minimizes the handoff and the packet delay. The TA is used with 6to4 Router, DSTM-TEP, ISTAP and NAT-PT. So, The TA supports transitions to mobile routers and mobile nodes as well as static hosts between IPv4 and IPv6 networks. In future work, we will implement and verify the proposed architecture of this paper.

\section{References}

1. D. Johnson, C. Perkins, J. Arkko, "Mobility Support in IPv6", IETF RFC 3775, June 2004.

2. IETF NEMO Working Group, "http://ietf.org/html.charters/nemo-charter.html".

3. V. Devarapalli, R. Wakikawa, A. Petrescu, P. Thubert, "Network Mobility (NEMO) Basic Support Protocol", IETF RFC 3963, January 2005.

4. T. Ernst, H-Y. Lach, "Network Mobility Support Terminology", draft-ietf-nemoterminology-04, October 2005.

5. C. Perkins, "IP Mobility Support for IPv4", IETF RFC 3220, January 2002.

6. H. Soliman, G. Tsirtsis, "Dual Stack Mobile IPv64", draft-soliman-v4v6-mipv4-02, July 2005.

7. P. Thubert, P. Molteni, P. Wetterwald, "IPv4 traversal for MIPv6 based Mobile Routers", draft-thubert-nemo-ipv4-traversal-01, May 2003.

8. R. Wakikawa, C. Williams, K. Uehara, J. Murai, "IPv4 Care-of Address Registration", draft-wakikawa-nemo-v4tunnel-01, February 2005.

9. J. Bound, L. Toutain, JL. Richier, "Dual Stack IPv6 Dominant Transition Mechanism (DSTM)", draft-bound-dstm-exp-04, October 2005.

10. G. Tsirtsis, P. Srisuresh, "Network Address Translation Protocol Translation (NAT-PT)", IETF RFC 2766, February 2000.

11. E. Nordmark, "Stateless IP/ICMP Translation Algorithm (SIIT)", IETF RFC 2765, February 2000.

12. N. Banerjee, "Mobility Support in Wireless Internet", IEEE Wireless Commun., vol.10, no.5, pp.54-61, October 2003. 